\title{
Estratificación de la reproducción y violencia obstétrica en servicios públicos de salud reproductiva*
}

\author{
ITZEL ADRIANA SOSA SÁNCHEZ**
}

\begin{abstract}
Stratified Reproduction and Obstetric Violence in Public Services of Reproductive Health. A qualitative investigation suggesting the social existence of coercive and tax practices -with different degrees of obstetric violenceas updating mechanisms of diverse hierarchies and social inequalities during the care of reproductive proceedings. These practices express a stratification of the reproduction, which tends to value certain practices and reproductive decisions (and maternities) as desirable or undesirable. Women's experiences in public services of reproductive health are an extension and an expression of broader experiences of structural violence, inequality and dominating relations.
\end{abstract}

Key words: sexual and reproductive rights, reproductive practices, contraception, childbirth, structural violence

\begin{abstract}
Resumen
Se presentan los resultados de una investigación cualitativa que sugieren la existencia de prácticas coercitivas e impositivas con distintos grados de violencia obstétrica como mecanismos de actualización de diversas jerarquías y desigualdades sociales durante la atención de eventos reproductivos. Estas prácticas expresan una estratificación de la reproducción que tiende a valorar determinadas prácticas y decisiones reproductivas (y maternidades) como deseables o indeseables. Las experiencias de las mujeres en los servicios públicos de salud reproductiva son la prolongación y expresión de experiencias más amplias de violencia estructural, desigualdad y relaciones de dominación. Palabras clave: derechos sexuales y reproductivos, prácticas reproductivas, anticoncepción, parto, violencia estructural
\end{abstract}

\section{Introducción}

$\mathrm{P}$ ese a la ratificación de tratados que protegen los derechos sexuales y reproductivos de las mujeres, y la implementación de programas con objeto de mejorar los servicios de salud reproductiva en México, varios estudios han documentado la persistencia de diversos problemas de salud reproductiva (Camarena y Lerner, 2008; Erviti, Sosa-Sánchez y Castro, 2010).

Se ha señalado el uso indiscriminado de intervenciones obstétricas (Suárez et al., 2012; Diniz y Chacham, 2004; Secretaría de Salud, 2011). Así, el empleo de cesáreas se ha elevado significativamente en todos los proveedores de servicios de salud (públicos y privados), ubicándose muy por encima del $15 \%$ de nacimientos por cesáreas recomendados por la Organización Mundial de la Salud (oms), tendencia que continúa a la alza

\footnotetext{
* Artículo recibido el 12/05/17 y aceptado el 20/07/17.

** Programa de Estudios de Población del Centro Regional de Investigaciones Multidisciplinarias de la Universidad Nacional Autónoma de México. Av. Universidad s/n, circuito 2, col. Chamilpa, Ciudad Universitaria de la uAEm, 62210, Cuernavaca, Morelos <itzela@correo.crim.unam.mx>. Organismos colaboradores de investigación: Universidad Laval, Fondo Henry Levesque, McNamara Memorial. ORCID: http:/ / orcid.org/0000-0002-1223-0829
} 
(Secretaría de Salud, 2011). Para 2012, el porcentaje de cesáreas efectuadas en la práctica pública oscilaba en $40.9 \%$ de los nacimientos, y en la práctica privada se ubicaba en 69.6\% (Suárez et al., 2012). Paralelamente, en México se considera que la episiotomía es la intervención quirúrgica más habitual en los servicios de salud y ha devenido una práctica rutinaria sin contar con suficiente sustento empírico sobre sus beneficios (Secretaría de Salud, 2011; Sadler et al., 2016).

Las evidencias sugieren la persistencia de prácticas médicas coercitivas e incluso de violencia obstétrica que vulneran los derechos sexuales y reproductivos de las mujeres en contextos institucionales (ValdezSantiago et al., 2013; Smith-Oka, 2009 y 2012; Erviti, Sosa-Sánchez y Castro, 2010). Si bien todas las usuarias de los servicios públicos de salud reproductiva están expuestas en mayor o menor grado tanto a las intervenciones médicas no justificadas, como a las prácticas médicas coercitivas y de maltrato, se ha indicado que las mujeres socialmente más desfavorecidas y que no encajan con el modelo activamente promovido por el Estado de "modernas" y "buenas madres" (Smith-Oka, 2012; Ellison, 2003; Erviti, Sosa-Sánchez y Castro, 2010) son con frecuencia objeto de estas prácticas, ${ }^{1}$ las cuales evidencian la estratificación de la reproducción prevaleciente en México.

Este concepto hace referencia al conjunto de políticas, prácticas y creencias que fomenta la reproducción biológica y las prácticas reproductivas de determinados grupos poblacionales (en general pertenecientes a las clases sociales dominantes), al tiempo que desincentiva e incluso estigmatiza la reproducción y las prácticas reproductivas de otras poblaciones (comúnmente pertenecientes a las clases sociales menos favorecidas o a minorías étnicas o raciales) (Harris y Wolfe, 2014). $\mathrm{Al}$ respecto, se ha sugerido el importante rol del Estado en esta estratificación, ya que éste, mediante diversas políticas públicas y campañas, promueve determinados discursos y normatividades sobre lo que son, en un momento histórico y social, las prácticas reproductivas (y de maternidad) deseables, "modernas" y apropiadas, y que expresan la estratificación de la reproducción dominante (Smith-Oka, 2009 y 2012; Ginsburg y Rapp, 1995). Así, las mujeres que no acatan la normatividad reproductiva vigente ${ }^{2}$ a través de sus prácticas y decisiones reproductivas son consideradas como malas madres e incluso malas ciudadanas (Smith-Oka, 2009 y 2012; Erviti, SosaSánchez y Castro, 2010), lo que condiciona las interacciones sostenidas entre las usuarias de los servicios de salud reproductiva y el personal de salud, repercutiendo en una atención diferencial de las usuarias (Erviti, Sosa-Sánchez y Castro, 2010) y, en ocasiones, en la vulneración de sus derechos sexuales y reproductivos (Smith-Oka, 2009 y 2012).

Lo antes mencionado ocurre en un contexto de marcada y creciente desigualdad social en México (Damián, Boltvinik y Marín, 2015), ${ }^{3}$ y de un complejo sistema de estratificación social y cívica que se articula con jerarquías de clase social, de generación y étnico-raciales (por mencionar algunas) (Navarrete, 2004), lo cual, en un país diverso y marcadamente desigual, adquiere consecuencias específicas sobre los derechos sexuales y reproductivos de las mujeres durante la atención de eventos reproductivos.

Este artículo explora el impacto de diversas desigualdades sociales y de los procesos de estratificación de la reproducción sobre los derechos sexuales y reproductivos de las usuarias de los servicios públicos de salud reproductiva en dos municipios del estado de Morelos.

\section{Metodología}

Se realizó una investigación sociológica-interpretativa en dos municipios del estado de Morelos: en barrios populares ${ }^{4}$ de la ciudad de Cuernavaca y el municipio

1 En México, la principal población objetivo de estas políticas públicas y campañas gubernamentales son las mujeres socialmente desfavorecidas, dado que es entre las mujeres pobres donde la tasa de fecundidad continúa siendo más elevada (Smith-Oka, 2009), y donde persiste un menor conocimiento de métodos anticonceptivos y un bajo uso de los mismos, así como un menor empleo de servicios biomédicos durante el parto (principalmente en mujeres pobres, indígenas y de residencia rural).

2 Tener pocos hijos, utilizar los servicios y obedecer prescripciones biomédicas (que incluye el uso de anticoncepción moderna), y alejarse de lo que se consideran prácticas reproductivas de alto riesgo.

3 En 2014, aproximadamente 84\% de la población en México vivía en situación de pobreza (y de él, más de 50\%, en pobreza extrema) (Damián, Boltvinik y Marín, 2015).

4 A diferencia de los estratos marginales, los populares acceden a diversos servicios (agua potable, línea telefónica, pavimento, etcétera), a viviendas de materiales durables, y tienen mayores oportunidades de empleo, por ejemplo, para los jefes de familia, sin que lleguen a contar con las comodidades de los sectores medios (Stern, 2003). Los barrios seleccionados para esta investigación presentan un perfil sociodemográfico típico de los barrios populares del centro de México y tienen una importante proporción de población de migrantes frecuentemente de origen indígena proveniente de Oaxaca y Guerrero; se ubican cerca de las zonas céntricas de Cuernavaca y de Tepoztlán y de los centros de salud donde se realizó el trabajo de campo. 
de Tepoztlán (en la cabecera y en Santa Catarina) en 2009 y 2010. Los lugares donde se llevó a cabo el trabajo de campo fueron seleccionados considerando que constituyen una muestra representativa de los barrios populares (de clase media-baja y baja) de la ciudad de Cuernavaca así como de las regiones rurales del centro de México. Se efectuó un muestreo intencional no probabilístico (Glaser y Strauss, 1967) y 30 entrevistas en profundidad (con una duración promedio de 75 minutos cada una) a partir de guías de entrevistas semiestructuradas hasta obtener la saturación teórica. Se entrevistó a 22 usuarias de los servicios de salud pública y a ocho informantes clave y se llevaron a cabo 80 horas de observación en las salas de espera en clínicas de la Secretaría de Salud. Los criterios iniciales de selección de las usuarias de los servicios de salud fueron la edad (tener entre 18 y 52 años), haber usado los servicios públicos de salud reproductiva en los últimos 12 meses y residir en los barrios elegidos para el estudio. Para los informantes clave, el principal criterio de selección fue estar relacionados con las clínicas o centros de salud en donde se realizó el trabajo de campo. Las entrevistas indagaron sobre significados, percepciones, creencias y valoraciones alrededor del cuerpo, la sexualidad y la reproducción, así como sobre las experiencias y percepciones de las participantes en torno a las interacciones sostenidas con el personal de salud (médicos y enfermeras), especialmente durante consultas relativas a la salud reproductiva (contracepción, embarazo, parto, aborto, etcétera). La selección de las localidades reconoce como relevante trabajar paralela y comparativamente con participantes de poblaciones urbanas y rurales asumiendo que el lugar de residencia conlleva una exposición diferencial a los discursos y prácticas médicas en torno a la reproducción y la sexualidad, lo que creemos tiene implicaciones en las interacciones sostenidas (y la percepción de las mismas) durante las consultas en salud reproductiva.

La mayoría de las participantes (usuarias) fue reclutada en centros de salud, en salas de consulta o en talleres del programa Oportunidade ${ }^{5}$ de la Secretaría de Salud. Siete de las participantes fueron contactadas con la técnica de bola de nieve (snow ball sampling). Se invitó de manera generalizada a las usuarias de los servicios públicos de salud a colaborar en el estudio. A las interesadas, se les aplicó un breve cuestionario sociodemográfico que permitió identificar a las potenciales participantes, privilegiando incluir sujetos con diversas características sociodemográficas consideradas centrales en la construcción de significados atribuidos al cuerpo, a la sexualidad y a la reproducción. ${ }^{6}$

Casi todas las entrevistas fueron audiograbadas. ${ }^{7}$ Con el propósito de cuidar rigurosamente los aspectos éticos del estudio, fue requisito indispensable obtener el consentimiento informado de las/los participantes. Se garantizó que la información proporcionada es por completo confidencial y usada para fines estrictamente de investigación. En la fase de análisis y difusión de resultados, toda identificación de las y los participantes fue anulada. Las entrevistas fueron transcritas de forma literal y sistematizadas, para proceder al análisis interpretativo mediante el software Atlas.ti. El examen de las narrativas privilegió el método propuesto por la teoría fundamentada (Glaser y Strauss, 1967), enfatizando en la comparación constante de los datos, y en un ir y venir entre los datos y la teoría.

\section{Características sociodemográficas \\ de la población participante \\ en el estudio}

a) Usuarias de los servicios de salud. La edad media de las 22 participantes en las entrevistas fue de 36.6 años; 15 residían en Cuernavaca y siete en Tepoztlán; 14 se consideraban católicas; 11 estaban unidas, siete solteras y cuatro divorciadas / separadas; 15 tenían hijos (dos hijos en promedio por mujer); siete contaban con licenciatura incompleta, siete con preparatoria/ carrera técnica, siete con primaria completa y una era analfabeta; cuatro se autodefinieron como indígenas, siete como mestizas y seis habitaban en un hogar indígena, sin embargo, la mayoría no supo cómo autoidentificarse étnico-racialmente; 15 eran económicamente activas y en su mayoría trabajaba en empleos no especializados (estilistas, trabajadoras de servicio doméstico, vendedoras, etcétera) y no contaba con seguridad social; y siete eran amas de casa. A partir de las condiciones generales de la vivienda (material

5 Oportunidades es un programa social de transferencia condicionada de dinero que desarrolla acciones intersectoriales para la educación, la salud y la alimentación, con la finalidad de promover el bienestar general de las familias de ingresos bajos o que viven en condiciones de pobreza alimentaria.

6 A saber: estado civil, edad, paridad (número de hijos), nivel de escolaridad, hablar una lengua indígena o provenir de un hogar indígena, y lugar de residencia.

7 A excepción de una entrevista con uno de los informantes clave que no consintió al audiograbado. 
del piso de la casa y hacinamiento por cuartos), ${ }^{8}$ los años de escolaridad y el lugar de residencia, se agrupó a las participantes en cuatro subgrupos para dar cuenta de sus condiciones objetivas de vida, obteniendo las siguientes combinaciones: contexto urbano con condiciones de vida precarias (cuatro participantes) o no precarias (11), y contexto rural con condiciones de vida precarias (seis) y no precarias (una). ${ }^{9}$

b) Informantes clave. La edad promedio fue de 46.6 años y tenían en promedio 2.8 hijos; cuatro trabajaban en Cuernavaca y cuatro en Tepoztlán; siete eran proveedores de servicios de salud (tres médicos, tres enfermeras, una promotora de salud y una partera); siete eran mujeres; cuatro eran solteros; tres se autodefinían como mestizos; y la mitad se negó a hablar de su pertenencia étnico-racial.

\section{Resultados}

\section{Los regaños y las bromas de carácter sexual durante la labor de parto}

En las narrativas de las mujeres participantes y de los informantes clave emerge continuamente la existencia de regaños y "bromas" hacia las mujeres en labor de parto por parte del personal de salud. En general, los discursos sugieren que los regaños son dirigidos fundamentalmente hacia las mujeres que no cumplen las expectativas de controlar el dolor (que no "cooperan" con el personal de salud) y expresan éste de manera poco tolerada (sobre todo gritando):

\section{—CCómo viviste las interacciones con el personal de salud? ¿Cómo te trataron? \\ - A mi lado estaban mujeres gritando y los doctores les decían: "Cállese, !ya cállese!”. Era muy violento [Ana, usuaria, 33 años, contexto urbano, condiciones de vida no precarias, no indígena].}

Si bien algunas participantes, como indica el testimonio de Ana, logran cuestionar estas prácticas e incluso conceptualizarlas como violentas o de maltrato (lo que sucede sobre todo con las más jóvenes y escolarizadas), en los discursos de la mayoría éstas emergen como justificables e incluso deseables:
- Algunas participantes comentan que, cuando gritan, las regañan en el área de Tococirugía, ¿te tocó ser testigo de este tipo de prácticas?

- Sí, sí me tocó verlo pero lo considero [silencio] un mal necesario, no precisamente que las regañen o que se burlen de ellas.

- ¿Las regañaban y se burlaban de ellas?

- Sí, porque en Tococirugía yo era la cama 20, jestaba lleno! Como puedes imaginar, 20, 19 estamos relax, las ves con su dolorcito, concentradas en lo que están haciendo, mientras unas parecen ambulancia y jahí sí!, las enfermeras tenían una actitud burlona, pero era con afán de relajar un poquito la tensión. Porque si una está ahí en su dolor y escuchas a una que sientes que se muere, te intimida mucho y puede empezar el descontrol con todas las mujeres. "Ya va a salir mi bebé!" "!Me voy a morir!", y las enfermeras, en tono burlón, le decían: "!Yo tengo tres chamacos y no me he muerto!", pero era de guasa [...] éramos tantas para dos médicos [Edith, usuaria, 33 años, contexto urbano, condiciones de vida no precarias, no indígena].

Las palabras de Edith sobre los regaños y las bromas (sobre este punto profundizaremos más adelante) del personal de salud dirigidos a las mujeres en labor de parto pone en evidencia dos cuestiones fundamentales. En primer lugar, visibiliza el grado de normalización de las usuarias de diversas formas de maltrato y violación de sus derechos sexuales y reproductivos en contextos institucionales, lo cual torna inteligible que estas prácticas de violencia ejercidas por el personal de salud sean percibidas por Edith (y por otras usuarias) como un "mal necesario" para evitar que las mujeres en labor de parto se descontrolen. Desde esta perspectiva, el rol social asignado a (y esperado en) las mujeres es el de autocontrolarse y cooperar (obedecer) con el personal de salud, y el de éste, garantizar que tal rol sea acatado por ellas $\mathrm{y}$, de no ser así, restablecer el control y el orden. Asimismo, el testimonio anterior muestra las condiciones poco favorables (las restricciones materiales y de recursos humanos) en las que el personal de salud atiende a las usuarias, lo cual ejerce presiones adicionales sobre él y repercute en el servicio prestado. De tal suerte, los testimonios de las participantes visibilizan una división simbólica (con consecuencias

8 El Instituto Nacional de Estadística y Geografía (2013) sugiere dos criterios para determinar la existencia de hacinamiento: el número de personas por cuartos totales que existen en la casa (excluyendo el baño) y por dormitorios. A partir de la información recabada durante el trabajo de campo de este estudio, la condición de hacinamiento fue construida con base en el número de cuartos totales en la vivienda.

9 Condiciones de vida no precarias: piso de la vivienda de loseta o azulejos, condición de hacinamiento baja y con estudios superiores a primaria. Condiciones de vida precarias: piso de la vivienda de cemento o terracería, condición de hacinamiento alta y con estudios de primaria completa o menos. 


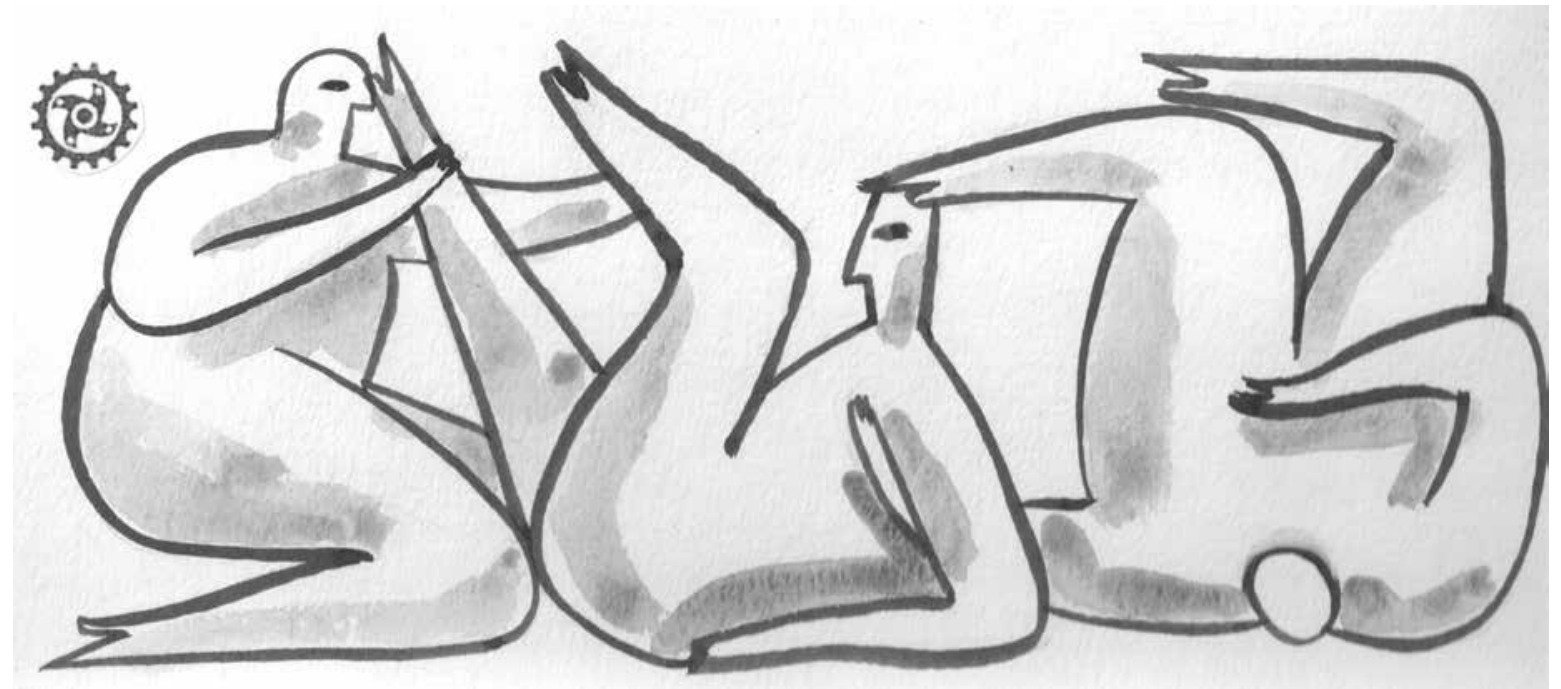

prácticas) de las mujeres en labor de parto, realizada por el personal de salud, lo que torna inteligible para éste y para las usuarias, el ejercicio de diversas prácticas de maltrato. En segundo lugar, sobresale la violencia hacia las mujeres a través de "bromas", principalmente de carácter sexual. Si bien en las narrativas éstas son presentadas con frecuencia como un mecanismo para relajar las situaciones e interacciones entre el personal de salud y las usuarias, el análisis de quién hace las bromas, hacia quién son orientadas, sobre qué versan, la relación entre los implicados, así como el momento en que éstas son expresadas, revela significativamente el lugar que ocupan en la estructura social (y de género) los participantes en las mismas:

Todavía recuerdo cuando le decía: “jay, doctor, me duele!” y dice: “iay, no, ¿y cuando estás abriendo las piernas?, ¿no es lo mismo?”. Y tú con el dolor, que te digan lo que sea [Lorenza, usuaria, 25 años, contexto urbano, condiciones de vida precarias, no indígena].

No es, pues, arbitrario que las mujeres y su sexualidad constituyan el objeto central de las bromas del personal de salud, evidenciando el orden altamente jerarquizado de la situación (quién está en la posición de poder y quién en la de subordinación). Siguiendo a Pizzini (1991), si consideramos que el humor en contextos institucionales de salud ginecológica tiene la función de reforzar las relaciones de poder existentes, esto implicaría que el propósito fundamental de estas bromas es actualizar las relaciones asimétricas de poder (las profesionales, de género, de generación, étnico-raciales, etcétera) durante las interacciones personal de salud-usuarias.

Además, aunque escasamente, en los testimonios de los informantes clave emergen también estas prác- ticas de maltrato y de violencia obstétrica hacia las usuarias:

La atención es muy frívola, muy despersonalizada, a mí me tocó ver cómo el médico trata groseramente a las mujeres. Me tocó mucho oír la expresión de "así como abriste las piernas con tanto gusto, ahora aguántate”. Ese tipo de expresiones machistas, muy insultantes hacia la mujer, cómo el médico macho, prepotente, llega a establecer sus reglas de juego con la mujer y hace las cosas a su manera sin que la mujer sepa realmente si la está atendiendo bien o mal, si el médico tiene razón o no, simplemente a someterse a la voluntad de un médico [Magda, informante clave, médica, 51 años].

Pese a que entre la mayoría del personal de salud las prácticas de violencia obstétrica hacia las usuarias no son vistas como tales y son presentadas como "normales", es relevante que el testimonio anterior las califique de groseras e insultantes y exprese la percepción de este tipo de violencia como una forma de machismo, poniendo de manifiesto el papel del personal de salud en la reproducción de las desigualdades de género, que si bien tienen lugar en entornos institucionales, prevalecen en la sociedad mexicana más allá de ellos.

\section{Estratificación de la reproducción y autonomía reproductiva en contextos altamente jerarquizados}

En las narrativas de los informantes clave emerge la dificultad de reconocer a las mujeres, pero sobre todo a las indígenas y a aquellas pertenecientes a clases sociales desfavorecidas, como sujetos autónomos y 
de derechos con capacidad plena de tomar decisiones libres e informadas sobre sus cuerpos y sus capacidades reproductivas. Es más, existe un cuestionamiento generalizado de sus decisiones reproductivas no sólo en los testimonios de los informantes clave sino en los de las usuarias, lo que hace patente la estratificación de la reproducción prevaleciente en México. Los testimonios de las/los participantes sugieren que los embarazos de las mujeres que reúnen ciertas características estigmatizadas (ser adolescente, pobre, con baja escolaridad, indígena, soltera, con alta paridad, o considerada "demasiado vieja o demasiado joven" para tener hijos) tienden a ser percibidos como un problema social que debe combatirse. Los discursos del personal de salud apuntan a que los esfuerzos tendientes a "convencer" a las pacientes para ser esterilizadas no son aleatorios y se dirigen prioritariamente hacia ciertas mujeres:

— ¿Cuándo se recomienda una отв (salpingoclasia)?

- Recomendamos las отв a las personas que ya tienen más de tres hijos, que son personas mayores, que vemos tienen bajos recursos y que no van a poder sobresalir [Rafael, informante clave, médico, 58 años].

Asimismo, los discursos del personal de salud aluden a una dificultad particular al trabajar con poblaciones indígenas bajo el supuesto (socialmente compartido) de una inferioridad cultural que tiene consecuencias sobre sus prácticas anticonceptivas y reproductivas:

Es muy difícil trabajar con indígenas, está la barrera de la lengua, además no usan anticonceptivos y se sientan en el piso, ;imagínese! [Flor, informante clave, enfermera, 40 años].

El testimonio anterior evidencia la circulación entre el personal de salud de los discursos que se desprenden de la estratificación de la reproducción dominante en la sociedad mexicana, lo que obliga a subrayar que la organización social de la sexualidad y de la reproducción está enmarcada en relaciones históricas de poder y jerarquías sociales de diversa índole, incluyendo las étnico-raciales. Así, en las narrativas de un importante número de usuarias, pero sobre todo de los informantes clave, surge la distinción entre una sexualidad animal caótica y no regulada y una controlada o civilizada. Incluso esta distinción se manifiesta en los regaños hacia las mujeres que no acatan la normatividad reproductiva:

Sí me dice [la enfermera] "coneja, como coneja con los hijos", pues es que yo también las pastillas no me las tomaba luego bien, como era el reglamento, también tengo la culpa, no me cuidé [Luz, usuaria, 52 años, contexto urbano, condiciones de vida precarias, indígena].

De tal suerte, no es casual que en los relatos de los informantes clave emerjan con frecuencia diversos aspectos de las prácticas cotidianas (y culturales) de los indígenas a través de los cuales se evalúa su grado de civilización. Estas descripciones y divisiones simbólicas (entre lo civilizado y lo no civilizado) expresan igualmente en distintos grados el racismo y las prácticas discriminatorias hacia las poblaciones indígenas (y afrodescendientes) prevalecientes en la sociedad mexicana. Este racismo se encuentra estructuralmente articulado no sólo con el clasismo sino con el sexismo y con la dominación masculina, lo que tiene consecuencias específicas en el ámbito de los derechos sexuales y reproductivos de las usuarias de los servicios de salud que son etnicizadas o racializadas. ${ }^{10}$ Tampoco es aleatorio que trabajar con poblaciones indígenas tienda a ser concebido por el personal de salud como "más difícil", ya que esta etiquetación y construcción de los grupos políticamente minoritarios permite controlarlos y justificar (percibir como necesario) esos controles. Este proceso de etiquetación, en el caso de los indígenas, es altamente etnicizado y racializado y se articula con otras desigualdades y estereotipos sociales:

\footnotetext{
- ¿Cómo ha sido su experiencia trabajando con población indígena?

- Con los indígenas sí es más difícil, porque de acuerdo a sus creencias dicen: "voy a tener los hijos que Dios me dé". La población que ya está más civilizada al contrario te dice: "sólo un hijo o dos porque la economía está dura, no hay trabajo, no hay qué comer". En cambio un indígena no, dice: "voy a tener los hijos que Dios me dé", no se preguntan cómo los van a mantener, tienen cuatro, cinco, y todavía quieren más [Alicia, informante clave, enfermera, 37 años].
}

\footnotetext{
${ }^{10}$ En este trabajo, la racialización y la etnicización son definidas, siguiendo a Massey (2008), como aquellos procesos de clasificación y jerarquización social basados en diferencias de origen étnico o racial, respectivamente. Estas clasificaciones son también formas de dominación que emplean las diferencias étnicas, culturales o raciales para estigmatizar y subordinar a ciertos grupos o individuos.
} 
En el testimonio anterior se manifiesta la perspectiva biomédica racionalista dominante, donde la gestión de la reproducción es central para evaluar el grado de civilización y de blanquitud ${ }^{11}$ ética y cultural de los individuos y de los grupos sociales. Desde esta perspectiva, en general el ethos de las mujeres socialmente desfavorecidas y sobre todo de las indígenas o aquellas con alta paridad (que son comúnmente aquellas etnicizadas y racializadas) se caracteriza para el personal de salud por su dificultad para programar el número y el momento de tener hijos. De tal modo, estas mujeres tienden a ser percibidas como más cercanas a la naturaleza y menos "civilizadas", legitimando que sean objeto de regaños y de la imposición (no exclusiva) de métodos anticonceptivos así como de otras prácticas coercitivas por parte del personal de salud.

Paralelamente y articulado con lo anterior, las narrativas muestran la fragilidad del consentimiento informado como mecanismo de salvaguarda de la autonomía reproductiva de las mujeres. Así, en los testimonios de las usuarias se pueden observar evidencias de prácticas coercitivas que condicionan su elección del método anticonceptivo y vulneran sus derechos sexuales y reproductivos. Si bien los testimonios sugieren que la información sobre anticoncepción tiende a ser presentada durante el control del embarazo, es principalmente en el posparto e incluso en las salas de parto (salas de expulsión) donde las mujeres se encuentran en situación de extrema vulnerabilidad y aislamiento, y donde el personal de salud, a través de diferentes mecanismos, les insiste en "decidirse" por un método anticonceptivo:

Normalmente a las mujeres, cuando están en el parto, sufriendo por el cuarto hijo, se les hace mucha insistencia [para operarse] [Rafael, informante clave, médico, 58 años].

Te preguntan si tienes planeado hacerte un control, qué vas a hacer, cuántos hijos tienes y obvio tratan de persuadirte de inmediato de que te operes o que te pongas algo. Lo que menos quieren son chamacos en el país, sí es muy insistente por parte de los médicos, de las enfermeras. La hoja que llenas es cuando ya vas con los dolores, ya estás adentro [Yazmin, usuaria, 35 años, condiciones de vida precarias, contexto urbano, indígena].
Estos testimonios visibilizan no sólo la existencia de prácticas coercitivas en momentos poco favorables para la toma de decisiones libres e informadas, sino cómo el contexto en el que estos "consentimientos" tienen lugar restringe de manera significativa las opciones reproductivas de las mujeres, habitualmente a la esterilización femenina o el dispositivo intrauterino (DIU), que son por lo general las más valoradas por el personal de salud.

- ¿Cómo decidiste emplear por primera vez el DIU?

- Porque exigen que te lo pongas cuando te vas a aliviar, te lo pones o te lo pones. [...] sí te preguntan pero, por decir, una chava que ha tenido seis hijos y no se lo quiere poner por la religión, van como unos diez doctores y le dicen: "señora, ¿se quiere llenar de hijos?”, o sea, está bien ¿no?, ya somos muchos en el mundo [Lorenza, usuaria, 25 años, contexto urbano, condiciones de vida precarias, no indígena].

Me pusieron luego luego el dispositivo. Me dijo: "para que puedas alimentar a tu bebé" [Sandra, usuaria, 26 años, contexto rural, condiciones de vida precarias, indígena].

En ocasiones, principalmente en el caso de mujeres con condiciones de vida precarias, el personal de salud les impone el método anticonceptivo (sobre todo el DIU) sin avisarles:

Nadie me preguntó, no me dijeron nada y me pusieron el dispositivo y yo ni en cuenta [...] después me hicieron una radiografía, y me dijo: "tienes un dispositivo, un DIU", dije: "pero, ¿cómo?, si no me lo puse", "te lo pusieron el día que nació tu bebé”, "pero, ¿cómo?, ni me dijeron, por eso no me lo revisé" [Sofía, usuaria, 36 años, contexto rural, condiciones de vida precarias, no indígena].

Estas prácticas expresan no sólo la preferencia del personal de salud por ciertos métodos anticonceptivos, sino también determinadas lógicas profesionales e institucionales que se encuentran enmarcadas en contextos sociales políticos e ideológicos prevalecientes en la sociedad en general y que exhiben una estratificación de la reproducción que condiciona qué prácticas reproductivas y maternidades son socialmente valoradas y promovidas y cuáles estigmatizadas $\mathrm{y}$, por tanto, qué cuerpos son el objeto privilegiado de

\footnotetext{
${ }^{11}$ Los estudios sobre el carácter racial de la blanquitud indican que ésta se conforma por un conjunto de dimensiones interconectadas que implican ventajas estructurales en las sociedades que han sido configuradas sobre una dominación étnico-racial. Se sugiere entonces problematizar la blanquitud visibilizando los mecanismos a través de los cuales se reproducen jerarquías sociales en las relaciones e interacciones sociales cotidianas, actualizando de esta manera el orden étnico-racial (Echeverría, 2007).
} 
controles a través de prácticas anticonceptivas y de esterilizaciones.

\section{Intervenciones obstétricas rutinarias en labor de parto}

Pese a las diversas recomendaciones de la oms relativas a la importancia de reducir las intervenciones médicas no necesarias durante la atención del parto y otros eventos reproductivos, los testimonios de las usuarias de los servicios de salud indican tanto la persistencia de este tipo de prácticas como su rutinización:

\section{- ¿Le hicieron episiotomía?}

- Sí.
- ¿Le explicaron por qué se iba a hacer la episiotomía? - No, en el Seguro Social se hace eso, creo, para evitar algún desgarre pero no lo consideran como algo que puedas decidir tú, es algo que ya se hace de rutina. Entonces, sí fue algo bien doloroso, la sutura. En ese momento, ni lo pienso, lo que quiero es que salga el bebé [Edith, usuaria, 33 años, contexto urbano, condiciones de vida no precarias, no indígena].

En ese momento a mí ni me dijeron nada, nada más sentí que estaba rasgada, calientísimo y que salió mi hija [Ana, usuaria, 33 años, contexto urbano, condiciones de vida no precarias, no indígena].

Estas narraciones revelan que la episiotomía no es presentada en los servicios públicos como una intervención obstétrica opcional y que las mujeres rara vez

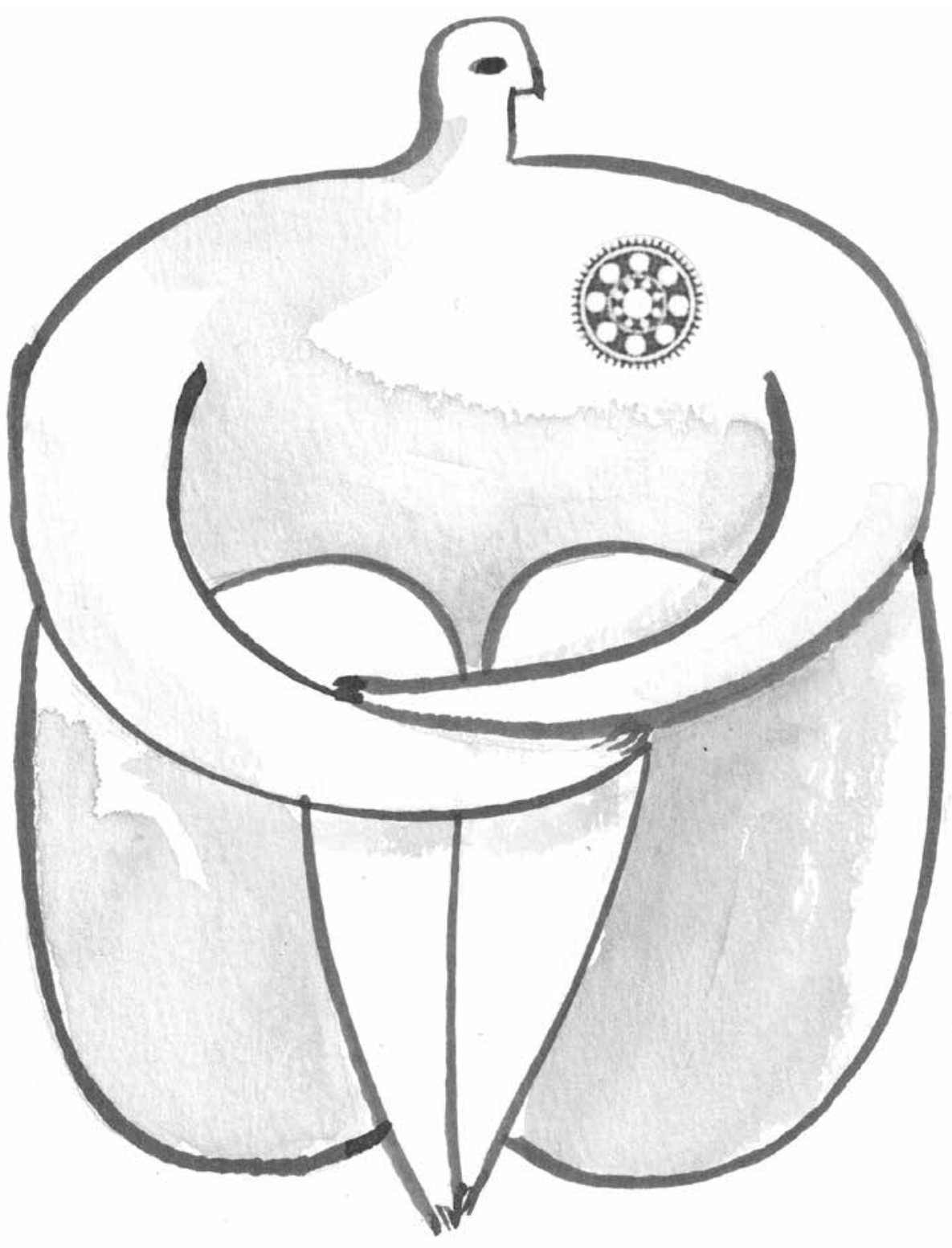


son advertidas de que serán sometidas a ella. Así, el consentimiento informado es pasado por alto la mayoría de las veces ante la realización de este procedimiento.

Paralelamente, en los discursos de las/los informantes clave, la episiotomía tiende a considerarse como un "mal menor", para prevenir el riesgo de un desgarro (sobre todo entre las mujeres primerizas):

La episiotomía es casi una regla en mujeres que van a tener su hijo por primera vez, por muchas razones. Una de ellas es que se supone que en el primer parto los tejidos no están tan elásticos, el riesgo de desgarre es muy alto. Entonces si no lo atiende una persona que tiene amplia experiencia, el riesgo de desgarre es muy alto [...]. Sí, hay un abuso de la episiotomía cuando se trata de partos por primera vez [Magda, informante clave, 51 años, médica].

No obstante en el discurso de la informante clave el riesgo de desgarro durante el parto de mujeres primerizas es expuesto como un hecho irrefutable, diversos trabajos han visibilizado la naturaleza política, problemática y no neutral de la evaluación y el manejo obstétrico de riesgos (Herr y Oaks, 2003).

Además de las razones de orden físico-anatómico que las/los informantes clave señalan para la rutinización de la episiotomía en las mujeres primerizas, otra es la falta de recursos humanos altamente capacitados disponibles en los servicios públicos de salud:

Sí se puede evitar, pero creo que para eso se necesita más supervisión y más cercanía con los pasantes, con los estudiantes de medicina, que están empezando sus primeros acercamientos con la atención de partos [Magda, informante clave, 51 años, médica].

En los testimonios de las usuarias también se hace patente la realización de tactos vaginales repetitivos que ellas algunas veces perciben como innecesarios y excesivos, sobre todo cuando sin consentimiento previo de la mujer en labor de parto se permite que los estudiantes de medicina aprendan (practiquen) con ella:

-Usted decía que la dejaron sufrir mucho. ¿Aqué se refiere?

- Me estuvieron haciendo el tacto a cada momento, a cada ratito, segundo por segundo. Entraba un doctor, otro, y otro, y otro, no sé si de por sí tenga que ser así que entren varios. Llegó el momento en que les dije: “¿Saben qué? Ya no me voy a dejar revisar, y si me muero, pues que me muera pero ya no, ya me acabaron de lastimar". Porque eran diferentes tipos de manos las que me tocaban, me dejaban muy lastimada, y luego ya no dejé al último que me tocara. Les contesté mal [Lety, usuaria, 48 años, contexto urbano, condiciones de vida precarias, no indígena].

$\mathrm{El}$ argumento anterior muestra que las usuarias tienen dificultades para reconocerse como sujetos de derechos y en general desconocen si pueden o no oponerse a determinadas prácticas (no sé si de por sí tenga que ser así), por lo que tienden a asumir que en contextos institucionales se espera de ellas obediencia (y sumisión), lo cual algunas veces es cuestionado (como en el caso de Lety) pese a las posibles repercusiones en el trato brindado por el personal de salud. Del mismo modo, como exhibe el testimonio siguiente, los discursos de las participantes con más de 35 años de edad, con condiciones de vida precarias, de zonas rurales y urbanas, revelan que sus expectativas se centran en salir vivas (no morirse) de sus estancias en el hospital (posteriores a la atención de partos, esterilizaciones, abortos, etcétera) y no en el reclamo por el reconocimiento de sus derechos a recibir servicios de salud de calidad en tiempo y forma:

- ¿Cómo la trataron?

- Pues yo digo que bien, digo, no me morí [Luz, usuaria, 52 años, contexto urbano, condiciones de vida precarias, indígena].

Es preciso señalar que estas expectativas están estrechamente relacionadas con las condiciones objetivas de vida de estas mujeres, en particular, y, en general, con un entorno de impunidad y de falta de reconocimiento de los derechos (no sólo reproductivos) en México.

Pese a lo antes mencionado, en los relatos de las mujeres menores de 35 años provenientes de contextos urbanos, con condiciones de vida no precarias, se alude de manera más abierta a los maltratos de los que fueron víctimas en los contextos institucionales durante la atención de diferentes eventos reproductivos:

- ¿Cómo te trataron en el hospital durante tus partos?

- Me trataron mal, esperé cinco horas afuera en plena calle, cuando entré era la una de la mañana. [...] me rasgaron, me lastimaron. Cuando salí estaba mi pierna negra, mi panza, porque se me recargó el doctor aquí para empujar al bebé con la rodilla [Lorenza, usuaria, 25 años, contexto urbano, condiciones de vida no precarias, no indígena].

Testimonios como el anterior indican que entre las mujeres más jóvenes (menores de 35 años) y con condiciones de vida no precarias el discurso sobre 
derechos ha comenzado a permear, lo que contribuye a que identifiquen con mayor facilidad (que las socialmente menos favorecidas y menos jóvenes) ciertas prácticas de violencia durante sus interacciones con el personal de salud, lo cual podría estar sugiriendo un mayor reconocimiento de sus derechos en este ámbito, quizá obedeciendo a una mayor difusión de estos derechos y a una toma de conciencia respecto al ejercicio y garantía de los mismos en ciertos contextos sociales menos desfavorecidos.

Asimismo, cabe apuntar, al igual que estudios como el de Erviti, Sosa-Sánchez y Castro (2010), que las preferencias anticonceptivas de los profesionales médicos están permeadas no sólo por las desigualdades sociales antes mencionadas (de género, clase social, afiliación étnico-racial, etcétera), sino también por la organización de los servicios de salud (la importante carga de trabajo, de horarios, etcétera) y la falta de recursos (humanos e infraestructura) en los servicios de salud pública, lo que además restringe y condiciona sus prácticas profesionales así como su preferencia de métodos anticonceptivos.

\section{A modo de reflexión final}

Los resultados muestran que la reproducción es un ámbito en el cual se manifiestan e intersectan desigualdades estructurales que condicionan no sólo la calidad del servicio ofertado, sino además el reconocimiento y respeto de los derechos sexuales y reproductivos de las mujeres e incluso sus derechos humanos.

Los derechos sexuales y reproductivos de las usuarias, sobre todo de aquellas provenientes de contextos sociales más desfavorecidos y de las que son en este contexto etnicizadas o racializadas, son con frecuencia vulnerados en el marco de las interacciones sostenidas entre las usuarias y el personal de salud. Los testimonios de las/los participantes sugieren la predominancia del modelo biomédico como la manera "ideal" de atender los eventos reproductivos, lo que incluye la rutinización de intervenciones como la episiotomía. Paralelamente, en las narrativas emerge la existencia de prácticas de violencia obstétrica que incluyen tanto la imposición o coerción para aceptar algún método anticonceptivo o esterilización, como regaños y bromas de carácter sexual en cuanto mecanismos de actualización de diversas jerarquías y desigualdades sociales (sobre todo, pero no exclusivamente, las de género) durante la atención de eventos reproductivos.
Si bien la imposición de métodos anticonceptivos y de esterilizaciones forzadas ha decrecido de manera significativa en las últimas tres décadas en México, esto no significa que hayan desaparecido, ni implica la ausencia de prácticas coercitivas y autoritarias en los contextos institucionales. Esto condiciona las decisiones reproductivas de las mujeres en los espacios de salud, vulnerando sus derechos sexuales y reproductivos, y visibiliza el peso de los contextos sobre sus decisiones, haciendo patente que en ocasiones el personal de salud induce o impone determinados métodos anticonceptivos sin dar espacio a la toma de decisiones libre e informada que un real consentimiento informado requiere como condición indispensable.

Los resultados manifiestan que no es aleatorio que sobre todo las mujeres socialmente más desfavorecidas (con menos escolaridad, de clase social más baja, etcétera) y que se alejan de las expectativas que norma el modelo reproductivo dominante (con más hijos, por ejemplo) son quienes tienden a experimentar estas prácticas impositivas de anticonceptivos (e incluso de esterilización) en los hospitales públicos en México. Son también estas mujeres muchas veces indígenas (y comúnmente etnicizadas o racializadas), solteras, con alta paridad, pobres, etcétera, las que en general son percibidas (y construidas) socialmente y por el personal de salud como irresponsables y poco capaces de controlar sus capacidades reproductivas. Estas prácticas expresan tanto la estratificación de la reproducción como una valoración social diferencial y jerarquizada de maternidad, y cuestionan la autonomía sexual y reproductiva de las mujeres dificultando su reconocimiento como sujetos plenos de derecho. Esto demuestra el carácter no sólo clasista y generizado sino altamente racializado y etnicizado de las interacciones entre el personal de salud y las usuarias de estos servicios. Así, las jerarquías étnico-raciales prevalecientes en la sociedad mexicana permean estas interacciones evidenciando la existencia de prácticas médicas en ocasiones discriminatorias e incluso racis$\operatorname{tas}^{12}$ que se manifiestan en mayor o menor medida en la gestión de la reproducción en contextos institucionales y espacios de salud con consecuencias por lo general negativas sobre los derechos sexuales y reproductivos de las usuarias. Esto visibiliza el importante rol de las instituciones y prácticas médicas en la legitimación y reproducción social no sólo de la estructura de clases sociales sino también de otras desigualdades sociales (como las étnico-raciales, de género, generacionales) (Waitzkin, 1991; Debb-Sossa, 2007).

\footnotetext{
${ }^{12}$ La ideología racista es un sistema de representación que se materializa en las instituciones sociales (a través de prácticas discriminatorias) y en las relaciones sociales organizando y dotando de sentido al mundo material y simbólico.
} 
Asimismo, los testimonios de las participantes muestran los déficits de capital (económico, simbólico, etcétera) de las mujeres frente al personal de salud, lo cual se intensifica ante el aislamiento y la situación de vulnerabilidad que conllevan la atención de algunos eventos reproductivos, restringiendo la toma de decisiones libre e informada en este ámbito, lo que contribuye al no reconocimiento e incluso a la violación de sus derechos sexuales y reproductivos.

Es preciso resaltar que las experiencias reproductivas de las mujeres, así como sus experiencias en los servicios públicos de salud durante la atención de eventos reproductivos son la prolongación y, al mismo tiempo, la expresión de experiencias más amplias de violencia estructural, relaciones de dominación (no sólo de género) y desigualdad social (Sosa-Sánchez y Menkes, 2015; Sadler et al., 2016; Smith-Oka, 2012 y 2015; Erviti, Sosa-Sánchez y Castro, 2010).

Al mismo tiempo, los resultados visibilizan el grado de normalización de las prácticas de deshumanización y de violencia ejercidas sobre las usuarias, tanto entre ellas como entre el personal de salud. Al respecto, la anuencia de las mujeres en defensa de la imposición de métodos anticonceptivos (y esterilizaciones femeninas) o de prácticas de violencia (que incluyen las prácticas autoritarias o coercitivas) se desprende de procesos de violencia simbólica (Bourdieu, 1997) que expresan no sólo la incorporación de la normatividad reproductiva dominante y la estratificación de la reproducción, sino el despliegue del conocimiento autoritario (authoritative knowledge) en los espacios de salud. Desde este conocimiento, los médicos son construidos como expertos, y la práctica médica, como una práctica neutra e incuestionable (Ellison, 2003; Sadler et al., 2016) y no como lo que es: una práctica social e histórica que expresa y reproduce valores, ideologías y discursos dominantes de la sociedad. Estos resultados son consistentes con las evidencias de diversos estudios realizados en México en la última década y que muestran desde diferentes ángulos esta problemática así como las consecuencias negativas de estas prácticas sobre la salud y los derechos sexuales y reproductivos de las mujeres usuarias de servicios públicos de salud (Valdez-Santiago et al., 2013; Erviti, Sosa-Sánchez y Castro, 2010; Sosa-Sánchez y Menkes, 2015; SmithOka, 2009, 2012 y 2015).

Sin embargo, es también notorio que, aunque sea someramente, los argumentos sobre equidad de género y de derechos comienzan a emerger en los discursos de las participantes (usuarias) más jóvenes (menores de 35 años) y provenientes de contextos menos desfavorecidos, lo cual puede plantear una ruptura generacional (por lo menos en ciertos contextos) en relación con una toma de conciencia relativa al ejercicio de los derechos sexuales y reproductivos entre las mujeres más jóvenes.

Finalmente, cabe destacar que las participantes pocas veces adoptan actitudes pasivas frente a las prácticas médicas. Así, aunque no incluidos en este análisis, algunos testimonios de las participantes revelan los complejos y a veces sutiles mecanismos a través de los cuales las mujeres cuestionan las prácticas opresivas y normativas a las que son confrontadas -pese a las posibles consecuencias negativas que esto les puede acarrear (por ejemplo, la intensificación de los regaños o de las actitudes hostiles y desaprobatorias por parte del personal médico)-, sin que esto signifique negar el carácter estructurado e institucional de las mismas, condicionando y restringiendo las opciones y el margen de acción de las mujeres en los espacios institucionales a un número reducido de opciones social y médicamente deseables y racionales.

\section{Fuentes}

Bourdieu, Pierre

1997 Méditations pascaliennes, Le Seuil, París, 316 pp.

Camarena, Rosa y Susana Lerner

2008 "Necesidades insatisfechas en salud reproductiva: mitos y realidades en el México rural", en Susana Lerner e Ivonne Szasz (coords.), Salud reproductiva y condiciones de vida en México, t. I, El Colegio de México, México, pp. 117-216.

Damián, Araceli, Julio Boltvinik

y AlEJANDRO MARÍN

2015 "Evolución de la pobreza y la estratificación social en México 2012-2014. Método de Medición Integrada de la Pobreza y la Estratificación Social (mмIPE)", El Colegio de México, México, 20 de julio <http://www.julioboltvinik.org/ images / stories / pobreza\%20presentacin $\% 20$ de\%20resultados\%202014\%20conferencia\% 20de\%20prensa.pdf $>$ [7 de julio de 2016].

DebB-Sossa, Natalia

2007 "Helping the 'Neediest of the Needy': An Intersectional Analysis of Moral-Identity Construction at a Community Health Clinic", en Gender \& Society, vol. 21, núm. 5, pp. 749-772.

Diniz, SiMONE

y Alessandra Chacham

2004 “'The Cut Above' and 'the Cut Below': The Abuse of Caesareans and Episiotomy in Sao Paulo, Brazil", en Reproductive Health Matters, vol. 12, núm. 23, pp. 100-110.

ECHEVERRÍA, BOLÍVAR

2007 "Imágenes de la blanquitud", en Diego Lizarazo et al., Sociedades icónicas. Historia, ideología y cultura en la imagen, Siglo xxi Editores, México, pp. 15-32.

Ellison, Marcia

2003 “Authoritative Knowledge and Single Women's Unintentional Pregnancies, Abortions, 
Adoption, and Single Motherhood: Social Stigma and Structural Violence", en Medical Anthropology Quarterly, vol. 17, núm. 3, pp. 322-347.

ERViti, JoAguina,

ITZel A. Sosa-SÁnchez

Y ROBERTO CASTRO

2010 "Social Origin of Contraceptive Counseling Practices by Male Doctors in Mexico", en Qualitative Health Research, vol. 20, núm. 6, pp.

GinsBurg, FAYE 778-787.

Y RAYNA RAPP

1995 Conceiving the New World Order: The Global Politics of Reproduction, University of California Press, Berkeley, 450 pp.

Glaser, Barney y Anselm Strauss

1967 The Discovery of Grounded Theory, Aldyne Gruyter Press, Nueva York, 284 pp.

HARRIS, L Y T. WOLFE

2014 "Stratified Reproduction, Family Planning Care and the Double Edge of History", en Curr Opin Obstet Gynecol., vol. 26, núm. 6, pp. 539-544.

Herr, Barbara y LAURY OAKS

2003 Risk, Culture and Health Inequality: Shifting Perceptions of Danger and Blame, Praeger, Westport, $241 \mathrm{pp}$.

Instituto Nacional de Estadística y Geografía

2013 Hombres y mujeres en México 2013, Instituto Nacional de Estadística y Geografía/Instituto Nacional de las Mujeres, México, 128 pp.

Massey, Douglas

2008 "La racialización de los mexicanos en Estados Unidos: estratificación racial en la teoría y en la práctica”, en Migración y Desarrollo, núm. 10, pp. 65-95.

Navarrete, Federico

2004 Las relaciones interétnicas en México, Universidad Nacional Autónoma de México, México,

Pizzini, Franca 145 pp.

1991 "Communication Hierarchies in Humor: Gender Differences in the Obstetrical/Gynaecological Setting”, en Discourse \& Society, vol. 2, núm. 4, pp. 477-488.

Sadler, Michelle, Mario Santos,

Dolores Ruiz-Berdún, Gonzalo Leiva,

Elena Skoko, Patricia Gillen

y Jette Clausen

2016 "Moving beyond Disrespect and Abuse: Addressing the Structural Dimensions of Obstetric Violence", en Reproductive Health Matters, vol. 24 , pp. 47-55.

Secretaría de Salud

2011 Observatorio del desempeño hospitalario 2011 , Subsecretaría de Integración y Desarrollo del Sector Salud-Secretaría de Salud, México, $155 \mathrm{pp}$.
SMith-OKa, VANIA

2009 "Unintended Consequences: Exploring the Tensions between Development Programs and Indigenous Women in Mexico in the Context of Reproductive Health", en Social Science \& Medicine, vol. 68, núm. 6, pp. 2069-2077.

SMith-OKa, VANia

2012 “They don't Know Anything. How Medical Authority Constructs Perceptions of Reproductive Risk among Low Income Mothers in Mexico", en L. Fordyce y A. Maraesa (eds.), Risk, Reproduction and Narratives of Experience, Vanderbilt University Press, Nashville, pp. 103-120.

SMith-OKa, VANia

2015 "Microaggressions and the Reproduction of Social Inequalities in Medical Encounters in Mexico", en Social Science \& Medicine, vol. 143, pp. 9-16.

SosA-SÁnchez, ITZEL A.

y Catherine Menkes

2015 “Como te ven te tratan': Desigualdades sociales en los espacios de salud reproductiva. Resultados de un estudio de caso en el centro de México", en Laura Rodriguez y Jhon Antón Sánchez (comps.), Situación de la población afro-descendiente e indígena en América Latina - puntos de reflexión para el debate sobre Cairo +20 , Asociación Latinoamericana de Población, Asociación Latinoamericana de Población (Serie e-investigaciones), Belo Horizonte, pp. $161-176$.

Stern, Claudio

2003 "Significados e implicaciones del embarazo adolescente en distintos contextos socioculturales de México: reseña de un proyecto en proceso", en Estudios Sociológicos, vol. XXI, núm. 63, pp. 725-745.

SuÁrez, Leticia, Lourdes Campero,

Elvia de la VARA, LEONOR Rivera,

María Isidra Hernández, Dilys Walker,

Mauricio Hernández y Eduardo Lazcano

2012 Elevada recurrencia a las cesáreas: revertir la tendencia y mejorar la calidad en el parto. Encuesta Nacional de Salud y Nutrición, 2012, Instituto Nacional de Salud Pública-Secretaría de Salud, México.

Valdez-Santiago, Rosario,

Elisa Hidalgo-SolóRzano,

MARIANA MoJARRO-IÑIgueZ

y Luz María Arenas-Monreal

2013 "Nueva evidencia a un viejo problema: el abuso de las mujeres en las salas de parto", en Revista CONAMED, vol. 18, núm. 1, eneromarzo, pp. 14-20.

WAITZKIN, HowARD

1991 The Politics of Medical Encounters. How Patients and Doctors Deal with Social Problems, Yale University Press, New Haven y Londres, 311 pp. 